\title{
Degree of improvement after hyperbaric oxygen therapy for compromised flap and graft: A case series.
}

\author{
Kamonwan Jenwitheesuk ${ }^{*}$, Ajanee Mahakkanukrauh², Wiyada Punjaruk ${ }^{3}$, Kriangsak Jenwitheesuk ${ }^{4}$, \\ Palakorn Surakunprapha1, Puttama Punyavong1, Kengkart Winaikosol', Bowornsilp Chowchuen', \\ Phasuk Limrattanapimpa ${ }^{5}$
}

${ }^{1}$ Plastic and Reconstructive Unit, Department of Surgery, Khon Kaen University, Khon Kaen, Thailand

${ }^{2}$ Division of Allergy-Immunology-Rheumatology, Department of Medicine, Khon Kaen University, Khon Kaen, Thailand

${ }^{3}$ Department of Physiology, Khon Kaen University, Khon Kaen, Thailand

${ }^{4}$ General Surgery Unit, Department of Surgery, Khon Kaen University, Khon Kaen, Thailand

${ }^{5}$ Hospital Hyperbaric Oxygen Therapy Unit, Srinagarind Hospital, Khon Kaen University, Khon Kaen, Thailand

\begin{abstract}
Objective: To determine the treatment outcome of hyperbaric oxygen therapy (HBOT) in compromised graft/flap.

Method: A retrospective study was conducted to identify patients who had undergone ischemic compromised graft and flap and received hyperbaric oxygen therapy at Srinagarind Hospital, Faculty of Medicine, Khon Kaen University, Thailand, between 2012 and 2017. Wound improvement was the primary outcome.

Results: Eleven patients with ischemic graft/flaps were enrolled in the study (9 adults; 2 children). The results showed that the treatment outcome for all patients undergoing skin flap/graft was improved. Patients classified as grade 3 and 4 (7 flaps in total) had improved wound healing without other adjunctive therapies whereas 4 patients-classified as grades 1 and 2-experienced partial wound healing and required surgical debridement with skin graft or flap reconstruction.

Conclusion: In flap or graft ischemia, HBOT represented an additional medical treatment that can enhance wound healing resulting in a satisfactory treatment outcome.
\end{abstract}

Keywords: Ischemic flap/graft, Hyperbaric oxygen therapy, HBOT.

Accepted on July 24, 2018

\section{Introduction}

Flap reconstruction is a surgery that closes a skin defect, which could not otherwise heal by skin graft alone. This is a complicated procedure and has various surgical plans for designing appropriate blood vessels to supply the flap. The most common unfavorable sequelae of flap reconstruction is ischemia of the flap, resulting in partial flap loss or total flap loss depending on the severity of the decreased blood supply. If the ischemia occurs after surgery, it is difficult to improve the blood supply to the flap. In the case of a skin graft, if the graft is thin and the wound bed is good with enough blood supply, the skin graft has a good chance of survival. Conversely, if the skin graft is thick or comprises various types of tissues (i.e., a composite graft), the chances of survival are low.

Currently, hyperbaric oxygen therapy (HBOT) has a crucial role in increasing oxygen supply to tissues and enhancing wound healing. It also has an influence on flap and graft salvage. Several previous studies revealed that HBOT provided important advantages in wound healing; it can be used alone to heal the wound or be combined with other therapies [1]. Examples of adjunctive therapy include HBOT plus (a) leech therapy, pentoxifylline, and topical nitroglycerin or (b) surgery $[2,3]$. The aim of the current study was to determine the treatment outcome of HBOT for compromised graft/flap.

\section{Materials and Methods}

This is a case series from Division of Plastic Surgery, Department of Surgery, the HBO Unit, Srinagarind Hospital, Faculty of Medicine, Khon Kaen University, Thailand. The inclusion criteria were all patients diagnosed as compromised graft and flap who received HBO therapy. The composite graft and flap defined as those with poor blood supply, evidence of ischemia, or composite graft/flap. The study period was 
between 2012 and 2017. Baseline clinical features, treatment options, HBO therapy (total oxygen time, number of dives) and results of wound healing of all eligible patients were recorded.

Details of all data of each individual were summarized. The main clinical outcome was improvement of wound healing which was classified into four grades as follows: grade 1having 1\%-25\% improved wound healing; grade 2-having 26\%-50\% improved wound healing; grade 3-having 51\%-75\% improved wound healing; and, grade 4-having 76\%-100\% improved wound healing.

The HBO protocol used in this setting was daily treatment with a 2-2.4 atmospheres absolute (ATA) for 60-90 $\mathrm{min}$ in a monoplace chamber. The number of treatment dives depended on patient compliance, patient tolerance, and response of the lesion.

The study was reviewed and approved by the Khon Kaen University Ethics Committee for Human Research (HE 611060).

\section{Results}

Over the study period, 270 patients were treated with HBOT at Srinagarind Hospital, but only 11 patients with compromised graft and flaps underwent HBOT were enrolled in the current study. Nine patients were adults and 2 were children. Most patients $(80 \%)$ were male

The first child patient was a 2-year-old boy, who was treated with replantation at the left leg, which was the first flap and the result of wound healing was not good so the wound defect persisted. The second flap, the anterolateral thigh free flap was consequently operated.

The second child patient was a 5-year-old boy with a compromised flap of the first dorsal metacarpal area. The clinical data and all variables of patients are presented in Table 1.

Table 1. Demographic data of patients who underwent hyperbaric oxygen therapy (HBOT) at Srinagarind Hospital between 2012 and 2017.

\begin{tabular}{|c|c|c|c|c|c|c|c|c|c|}
\hline No. & Age & Sex & Compromised graft/flap & Dives & $\begin{array}{l}\text { Grade of } \\
\text { improvement }^{*}\end{array}$ & Combined Tx & $\begin{array}{l}\text { Pre HBOT } \\
\mathrm{O}_{2}\end{array}$ & $\begin{array}{l}\text { Post HBOT } \\
\mathrm{O}_{2}\end{array}$ & $\begin{array}{l}\text { HBOT on } \\
\text { post op day }\end{array}$ \\
\hline 1 & 2 & M & Replantation left leg & 30 & 2 & Multiple debridement & NA & NA & 6 \\
\hline 2 & 2 & M & Pre-op anterolateral thigh flap & 10 & 4 & No & NA & NA & Pre-op \\
\hline 3 & 63 & $\mathrm{~F}$ & Pectoralis major flap & 30 & 2 & Debridement, flap & 98 & 100 & 8 \\
\hline 4 & 14 & M & Separated $2^{\text {nd }}$ and $3^{\text {rd }}$ toe & 20 & 1 & Debridement, FTSG & 98 & 100 & 6 \\
\hline 5 & 69 & M & Supraclavicular flap & 20 & 2 & Debridement, STSG & 98 & 100 & 4 \\
\hline 6 & 35 & $\mathrm{~F}$ & Composite graft & 9 & 4 & No & 98 & 100 & 2 \\
\hline 7 & 24 & M & Composite graft & 10 & 4 & No & 98 & 100 & 2 \\
\hline 8 & 5 & M & $1^{\text {st }}$ dorsal metacarpal flap & 10 & 3 & $2^{\text {nd }}$ healing & NA & NA & 4 \\
\hline 9 & 48 & M & Thoracodorsal perforater flap & 10 & 4 & No & 98 & 100 & 3 \\
\hline 10 & 18 & M & Composite graft & 10 & 4 & No & 98 & 100 & 3 \\
\hline 11 & 18 & M & $1^{\text {st }}$ dorsal metacarpal flap & 10 & 4 & No & 98 & 100 & 3 \\
\hline
\end{tabular}

Note. FTSG: Full-thickness skin grafts; Tx: Treatment; NA: Not Available; ' grade of improvement: grade 1: improve<25\%, grade 2: improve 25\%-49\%, grade 3: improve $50 \%-74 \%$, grade 4 : improve $75 \%-100 \%$.

There was improvement of wound healing as follows: grade 1 flap (1 patient), grade 2 (3 patients), grade 3 (1 patient) and grade 4 grafts/flaps ( 6 patients). Patients having grades 3 and 4 (7 flaps in total) had improved wound healing without other adjunctive therapies whereas the 4 patients with grades 1 and 2 had partial wound healing and required surgical debridement with skin graft or flap reconstruction.

\section{Discussion}

Ischemia or insufficient blood supply is the key limitation in wound healing. So, enhancing oxygen supply to the wound is essential for improved wound healing. Oxygen has important roles in improving wound healing through (a) increasing the number of fibroblasts, (b) enhancing collagen synthesis, (c) stimulating angiogenesis, (e) increasing keratinocyte differentiation, (f) re-epithelialization, and (g) oxidative killing of bacteria [4-6].

Numerous surgical procedures require graft and flap for treatment of wound healing. Generally, the flap is used for treatment of wound defect-a random or axial pattern. The most common complication of the flap is flap ischemia. Thus, all surgeons need to be proactive about preventing flap ischemia in their surgical planning. Using other medical equipment can help mitigate flap ischaemia-i.e., doppler ultrasound [7], CT angiogram [8,9], and magnetic resonance angiography [10]; by helping to determine the location of vessels supplying the 
lesion when it is uncertain whether the vessels were damaged after trauma.

Four factors play important roles in enhancing good surgical outcome vis-à-vis wound healing, including (a) good wound bed preparation, (b) meticulous surgical technique, (c) stabilization of graft, and (d) prevention of hematoma. Wound bed preparation is essential for preparing the wound prior to surgery in order to close the wound with the flap. This preparation can effectively decrease the risk of infection, which can lead to flap ischemia. The healing process of a graft is basically different from a flap because there are no blood vessels directly supplying the graft. The graft receives all necessary nutrients from plasmatic imbibition.

Notwithstanding meticulous technique during surgery flap complications still occur. Generally, if a surgical problem is detected during surgery, the flap is removed back to the origin or a delayed or supercharge technique is performed. The latter increases blood supply to the flap [11] or augments venous outflow [12]. If flap ischemia is detected after surgery, the causes of ischemia are corrected when found (i.e., thrombosis of blood vessel). If the cause of flap ischemia is unknown or known causes are detected but re-operation would not help to promote blood supply to the ischemic flap, a surgical approach is postponed. When hematoma or displacements of graft are detected at an early stage, the problem can generally be resolved. A composite graft, by comparison, is thick and it is necessary to preserve the thickness, but these results in an elevated risk of ischemia leading to poor surgical outcomes.

HBOT is an alternative treatment for patients with flap or graft ischemia. Many mechanisms of HBOT have been proposed to promote salvage of the graft and flap, including (a) enhancing angiogenesis [13], (b) decreasing swelling [14], (c) improving the immune response [14], and (d) preventing reperfusion injury [15]. Previous studies on HBOT outcomes for treating flap ischemia in animal studies were positive: HBOT significantly promoted survival of flap [14-16]. Clinical studies had no control group, had a small number of patients in the control group, or were case reports or case series. Some studies supported the hypothesis that HBOT promoted wound healing [17] while some revealed that HBOT outcomes did not differ significantly from conservative treatment [18].

The criteria for using HBOT in patients prior to surgery are having (a) a high risk of ischemia (i.e., exposure to radiotherapy), (b) a history of flap necrosis from a previous surgery, or (c) other factors that might influence wound healing (i.e., infection) [19]. HBOT effectively helped to promote wound healing in flap ischemia when it was used to treat patients as early as possible; especially when patients were treated with HBOT after a few days of ischemia [20].

HBOT is still the most suitable choice for the treatment of patients with an unknown cause of flap ischemia or the cause of ischemia is not vascular occlusion, which can be corrected by surgery. Thus, when the problem in the flap is not ischemia, HBOT is not necessarily used to prevent flap ischemia. If, however, surgeons planned to use HBOT in patients without flap ischemia, others factors should be considered (i.e., the cost and effectiveness of treatment, the length of hospital admission, and delayed surgery. To date, there has been no study revealing the results of treatment effectiveness and compare all those factors in using HBOT in patients without flap ischemia.

A current review showed that HBOT is beneficial in several flap settings including dog bite, nasal defect, replantation, or war injury reconstruction [21]. The HBOT should be treated as early as possible particularly within three days after surgery [22]. This study added information on HBOT on compromised flap/graft as follows: a) HBOT can be used successfully in children. Two children with compromised flap/replantation were improved with HBOT (Patients no. 1 and 2). b) HBOT can be used pre-operatively and with or without surgical intervention. A child had successfully treated with the HBOT was used pre-operative without any other combination treatment (Patient no. 2). c) HBOT can be used in all areas of the body. The HBOT therapy in this case series was performed from head to toe. d) HBOT is still beneficial even after three days of surgery (Patients no. 1, 3, 4, 5, 8). e) The overall successful rate (grades 3 and 4 improvement) was $7 / 11$ patients $(63.6 \%)$.

The limitations of this study follow. This was a retrospective study. For example, the clinical data all for patients was not all recorded, so further studies are needed. Another limitation was that we should not randomize the control trial. To study the treatment outcome of HBOT in patients with flap ischemia compared to the conventional treatment. However, a randomize control trial cannot be used because all patients with flap ischemia should have a chance to receive additional therapy such as HBOT. Even if the treatment outcome of HBOT will only slightly promote wound healing, all surgeons should consider this protocol so as to preserve the flap for survival. Patients with an ischemia flap could not be randomized resulting in no control group of patients with no HBOT.

\section{Conclusion}

In flap or graft ischemia, HBOT represents an additional medical treatment that can enhance wound healing, resulting in improved treatment outcomes.

\section{Conflict of Interest}

None

\section{Acknowledgements}

The authors wish to thank Mr. Bryan Roderick Hamman for assistance with the English-language presentation of the manuscript under the aegis of the Publication Clinic, Research Affairs, Faculty of Medicine, KKU. The authors also would like to thank Sleep Apnea Research Group, Research Center in Back, Neck and Other Joint Pain and Human Performance, Research and Training Center for Enhancing Quality of Life of Working Age People, Khon Kaen University for their kind support. 


\section{References}

1. Kucur C, Durmus K, Uysal IO, Old M, Agrawal A, Arshad H, Teknos TN, Ozer E. Management of complications and compromised free flaps following major head and neck surgery. Eur Arch Otorhinolaryngol 2016; 273: 209-213.

2. Moffat AD, Weaver LK, Tettelbach WH. Compromised breast flap treated with leech therapy, hyperbaric oxygen, pentoxifylline and topical nitroglycerin: a case report. Undersea Hyperb Med 2015; 42: 281-284.

3. Baynosa RC, Zamboni WA. The effect of hyperbaric oxygen on compromised grafts and flaps. Undersea Hyperb Med 2012; 39: 857-865.

4. Kuffler DP. Hyperbaric oxygen therapy: an overview. J Wound Care 2010; 19: 77-79.

5. Rodriguez PG, Felix FN, Woodley DT, Shim EK. The role of oxygen in wound healing: a review of the literature. Dermatol Surg 2008; 34: 1159-1169.

6. Jonsson K, Jensen JA, Goodson WH, Scheuenstuhl H, West J, Hopf HW, Hunt TK. Tissue oxygenation, anemia, and perfusion in relation to wound healing in surgical patients. Ann Surg 1991; 214: 605-613.

7. Debelmas A, Camuzard O, Aguilar P, Qassemyar Q. Reliability of color Doppler ultrasound imaging for the assessment of anterolateral thigh flap perforators: a prospective study of 30 perforators. Plast Reconstr Surg 2018; 141: 762-766.

8. Shen Y, Huang J, Dong MJ, Li J, Ye WM, Sun J. Application of computed tomography angiography mapping and located template for accurate location of perforator in head and neck reconstruction with anterolateral thigh perforator flap. Plast Reconstr Surg 2016; 137: 1875-1885.

9. Ono S, Hayashi H, Ohi H, Ogawa R. Imaging studies for preoperative planning of perforator flaps: an overview. Clin Plast Surg 2017; 44: 21-30.

10. Schaverien MV, Ludman CN, Neil-Dwyer J, Perks AG, Raurell A, Rasheed T, McCulley SJ. Relationship between venous congestion and intraflap venous anatomy in DIEP flaps using contrast-enhanced magnetic resonance angiography. Plast Reconstr Surg 2010; 126: 385-392.

11. Zhang $\mathrm{Y}$, Wang $\mathrm{T}$, Wei J, He J, Wang T, Liu Y, Xu H, Dong J. Whats the remedy for the distal necrosis of DIEP flap, better venous drain or more arterial supply? PLoS One 2017; 12: 0171651.

12. Ochoa O, Pisano S, Chrysopoulo M, Ledoux P, Arishita G, Nastala C. Salvage of intraoperative deep inferior epigastric perforator flap venous congestion with augmentation of venous outflow: flap morbidity and review of the literature. Plast Reconstr Surg Glob Open 2013; $1: 52$.
13. Liu X, Yang J, Li Z, Yang L, Wang C, Gao C, Liang F. Hyperbaric oxygen preconditioning promotes neovascularization of transplanted skin flaps in rats. Int $\mathrm{J}$ Clin Exp Pathol 2014; 7: 4734-4744.

14. Kahle AC, Cooper JS. Hyperbaric, physiological and pharmacological effects gases. StatPearls (Internet). Treasure Island (FL): StatPearls Publishing 2018.

15. Kang N, Hai Y, Liang F, Gao CJ, Liu XH. Preconditioned hyperbaric oxygenation protects skin flap grafts in rats against ischemia/reperfusion injury. Mol Med Rep 2014; 9: 2124-2130.

16. Fernando P. da Rocha, Djalma J. Fagundes, Jefferson A. Pires, Fernanda Salim T. da Rocha. Effects of hyperbaric oxygen and $\mathrm{N}$-acetylcysteine in survival of random pattern skin flaps in rats. Indian J Plast Surg 2012; 45: 453-458.

17. Copeland-Halperin LR, Bruce SB, Mesbahi AN. Hyperbaric oxygen following bilateral skin-sparing mastectomies: a case report. Plast Reconstr Surg Glob Open 2016; 4: 680.

18. Shuck J, OKelly N, Endara M, Nahabedian MY. A critical look at the effect of hyperbaric oxygen on the ischemic nipple following nipple sparing mastectomy and implant based reconstruction: a case series. Gland Surg 2017; 6: 659-665.

19. Bowersox JC, Strauss MB, Hart GB. Clinical experience with hyperbaric oxygen therapy in the salvage of ischemic skin flaps and grafts. J Hyperbaric Med 1986; 1: 141-149.

20. Quirinia A, Viidik A. The effect of hyperbaric oxygen on different phases of healing of ischaemic flap wounds and incisional wounds in skin. Br J Plast Surg 1995; 48: 583-589.

21. Francis A, Baynosa RC1. Hyperbaric oxygen therapy for the compromised graft or flap. Adv Wound Care (New Rochelle) 2017; 6: 23-32.

22. Roje Z, Roje Z, Eterovic D, Druzijanic N, Petricevic A, Roje T, Capkun V. Influence of adjuvant hyperbaric oxygen therapy on short-term complications during surgical reconstruction of upper and lower extremity war injuries: retrospective cohort study. Croat Med J 2008; 49: $224-232$.

\section{*Correspondence to}

Kamonwan Jenwitheesuk

Plastic and Reconstructive Unit

Department of Surgery

Khon Kaen University

Khon Kaen

Thailand 